Sirman Ya. V., Savytskyi I. V. Analysis of peroxidase activity in diabetic retinopathy and in applying various corrective means. Journal of Education, Health and Sport. 2019;9(11): 442-454. eISSN 2391-8306. DOI http://dx.doi.org/10.12775/JEHS.2019.09.11.041 https://apcz.umk.pl/czasopisma/index.php/JEHS/article/view/JEHS.2019.09.11.041

http://dx.doi.org/10.5281/zenodo.4498977

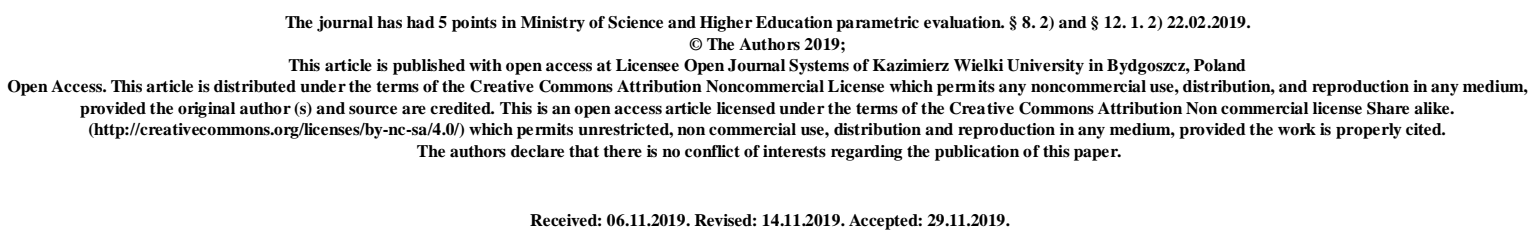

УДК:616-06:616-092.9

\title{
ANALYSIS OF PEROXIDASE ACTIVITY IN DIABETIC RETINOPATHY AND IN APPLYING VARIOUS CORRECTIVE MEANS
}

\author{
Ya. V. Sirman ${ }^{1}$, I. V. Savytskyi ${ }^{2}$
}

${ }^{1}$ SE "Ukrainian Research Institute of Transport Medicine of the Ministry of Health of

\author{
Ukraine"
}

\section{${ }^{2}$ Odessa International Medical University}

Sirman Yana Vadymivna, Candidate of Medical Sciences, Senior Researcher, Laboratory of Occupational Pathology, SE "Ukrainian Research Institute of Transport Medicine of the Ministry of Health of Ukraine"; http://orcid.org/0000-0002-9754-2564; yanasirman@ gmail.com

Savitsky Ivan Volodymyrovych, Doctor of Medical Sciences, Professor, Head of the Department of Medical and Biological Sciences of Odessa International Medical University; https://orcid.org/00000002-5841-9993; farmakod@ukr.net

For correspondence: Savitsky Ivan Volodymyrovych, 65039, Odessa, Fontanska doroha 4-a, kv.29, tel. + 38050-381-21-83, e-mail-farmakod@ukr.net

\section{Abstract}

Hyperglycemia stimulates the development of oxidative stress, which in turn is a powerful pathophysiological mechanism for the development of microvascular complications in diabetes. Increased production of reactive oxygen species is observed both during development and during the progression of diabetic retinopathy.

The study was performed on white Wistar rats weighing 180-200 g. According to the tasks, the animals were divided into 7 groups: 1st group - 60 intact animals; Group 2 - 60 
animals in which diabetic retinopathy was simulated without further correction. Group 3 - 60 animals, which simulated diabetic retinopathy with subsequent correction of hyperglycemia; Group 4 - 60 animals in which diabetic retinopathy was simulated with subsequent correction of hyperglycemia, administration of aflibercept and L-arginine solution; Group 5 - 60 animals in which diabetic retinopathy was simulated with subsequent correction of hyperglycemia, administration of aflibercept and bromfenac; Group $6-60$ animals in which diabetic retinopathy was simulated with subsequent correction of hyperglycemia, administration of aflibercept, L-carnitine and bromfenac; Group 7 - 60 animals, which simulated diabetic retinopathy with subsequent correction of hyperglycemia, the introduction of aflibercept, a solution of L-arginine and citicoline.

The results indicate the development of oxidative stress from the $30^{\text {th }}$ and with subsequent progression on the $60^{\text {th }}$ and $180^{\text {th }}$ days of experimental diabetic retinopathy, which is confirmed by a decrease in peroxidase activity in the $2^{\text {nd }}$ group, the maximum of which is observed in the $3^{\text {rd }}$ stage. Correction with hypoglycemic agents in group 3 had a positive effect, but was not able to restore the activity of the antioxidant enzyme, so there was a need for additional drugs. The use of aflibercept and nitric oxide donor in group 4 to correct the development of diabetic retinopathy had a positive effect on increasing the activity of peroxidase, which peaked on the $180^{\text {th }}$ day of the experiment, but did not reach the control values. The combined administration of bromfenac and aflibercept in group 5 was shown to significantly increase antioxidant activity, but not as significantly as in group 4. Administration of aflibercept, L-carnitine, and bromfenac to group 6 animals was shown to restore antioxidant protection as early as day 30 and was continued on days 60 and 180 of the study, but the results did not reach control values. The combination of metformin, aflibercept, L-arginine and citicoline in rats of the $7^{\text {th }}$ group proved to be the most effective correction, as evidenced by the normalization of peroxidase activity on the $30^{\text {th }}$ and $60^{\text {th }}$ day of the experiment, and on the $180^{\text {th }}$ recovery of marker activity to control values was recorded.

Keywords: experimental diabetic retinopathy; oxidative stress; antioxidants; peroxidase; correction; metformin; aflibercept; L-arginine; citicoline; L-carnitine; bromfenac.

\section{Introduction}

Diabetes mellitus (DM) is an urgent problem of the XXI century through the continuous progression of the incidence of both types 1 and 2 of the disease [1]. It should be emphasized that according to the WHO, diabetes mellitus is considered a pandemic of the $21^{\text {st }}$ 
century. In 2017, according to the International Diabetes Federation (IDF), the number of patients with diabetes mellitus is 424.9 million worldwide, which is a worldwide problem for humanity [2]. Macro- and microvascular complications significantly worsen the course of diabetes mellitus and cause the pathogenesis of endothelial dysfunction [2].

Hyperglycemia stimulates the development of oxidative stress, which in turn is a powerful pathophysiological mechanism for the development of microvascular complications in diabetes mellitus [3]. Enhanced production of reactive oxygen species is observed both during the development and progression of diabetic retinopathy. Oxidative stress (OS) underlies the pathogenesis of both insulin resistance and vascular diabetic complications, in particular diabetic retinopathy [4]. With the development of OS, which increases during the toxic effect of hyperglycemia on metabolic processes, an increase in the number of free radicals and inhibition of the antioxidant defense system is observed [5].

It has been proven that oxidative stress not only contributes to the development of retinopathy, but maintains this pathological condition even with normalization of glucose levels $[4,6]$. Decreased retinal activity of antioxidant enzymes such as glutathione reductase, glutathione peroxidase, superoxide dismutase and catalase, observed in diabetes, also contribute to the development of oxidative stress [7, 8]. According to one hypothesis, oxidative stress in diabetic retinopathy is a link between metabolic pathways that have been damaged by hyperglycemia. Reactive oxygen species, which are formed in mitochondria, destruct DNA, and thus activate poly-ADP-ribose polymerase [4].

The aim of the study: analysis of changes in blood peroxidase activity in experimental diabetic retinopathy and various methods of its correction.

Materials and methods. The study was performed on white Wistar rats weighing 180-200 g. According to the tasks, the animals were divided into 7 groups:

Group 1 - 60 intact animals;

Group 2 - 60 animals in which diabetic retinopathy was simulated without further correction.

Group 3 - 60 animals in which diabetic retinopathy was simulated with subsequent correction of hyperglycemia.

Group 4 - 60 animals, which simulated diabetic retinopathy with subsequent correction of hyperglycemia, administration of aflibercept and L-arginine solution.

Group 5 - 60 animals, which simulated diabetic retinopathy with subsequent correction of hyperglycemia, administration of aflibercept and bromfenac. 
Group 6 - 60 animals in which diabetic retinopathy was simulated with subsequent correction of hyperglycemia, administration of aflibercept, L-carnitine and bromfenac.

Group 7 - 60 animals, which simulated diabetic retinopathy with subsequent correction of hyperglycemia, the introduction of aflibercept, a solution of L-arginine and citicoline.

Type 2 diabetes mellitus and diabetic retinopathy were simulated by intraperitoneal administration of streptozotocin (Sigma, USA) dissolved in $0.1 \mathrm{M}$ citrate buffer with a $\mathrm{pH}$ of 4.5 [9]. The dose of streptozotocin $55 \mathrm{mg} / \mathrm{kg}$ body weight was divided into two injections. The introduction of streptozotocin was preceded by a high-fat diet for 28 days.

Doses of drugs:

Hypoglycemic medications - metformin (Merck Sante, manufacture France) - in a dose of $300 \mathrm{mg} / \mathrm{kg}$ in drinking form [10] $0.9 \%$ sodium chloride solution via syringe with intragastric probe daily.

Administration of L-arginine solution, which is a donor of NO, (SIMESTA, made in China, USP32 quality standard) was carried out by intragastric administration of L-arginine solution in $0.9 \%$ sodium chloride solution at a dose of $500 \mathrm{mg} / \mathrm{kg}$ [11] through a syringe with intragastric tube. The volume of the solution depended on the weight of the animal and did not exceed $1 \mathrm{ml}$. The drug was administered once a day before morning feeding, daily for 10 days [11].

Aflibercept (anti-VEGF therapy) was administered as a subconjunctival injection at a dose of $0.08 \mathrm{ml}(25 \mathrm{mg} / \mathrm{ml})$ [12].

Bromfenac - instillation of $0.09 \%$ solution of eye drops once a day.

L-carnitine (Sigma, USA) was administered in the form of an aqueous solution through a syringe with an intragastric tube at a dose of $25 \mathrm{mg} / 100 \mathrm{~g}$ of animal weight [13, 14].

Citicoline $-81.8 \mathrm{mg} / \mathrm{kg}(0.33 \mathrm{ml} / \mathrm{kg})$ was administered intramuscularly once a day.

Withdrawal of animals from the experiment was carried out in three stages:

$1^{\text {st }}$ stage of the study - the $30^{\text {th }}$ day after the start of modeling diabetes mellitus;

$2^{\text {nd }}$ stage of the study - the $60^{\text {th }}$ day after the start of modeling diabetes;

$3^{\text {rd }}$ stage of research - the $180^{\text {th }}$ day after the simulation diabetes.

Animals were removed from the experiment by decapitation under light ether anesthesia in accordance with the "Rules for performing work using experimental animals", approved by the Order of the Ministry of Health of Ukraine № 249 from 01.03.2012 and the Law of Ukraine № 3447-IV "On protection of animals from cruelty" (as amended from 15.12.2009 and from 16.10.2012). 
Blood was taken from the retroorbital venous plexus, which lies in orbit behind the eyeball. The puncture was performed in a circular motion with a glass pipette with an extended capillary, the tip of which is ground at an angle of $45^{\circ}$. A conjunctival sac was punctured in the medial corner of the eye between the eyeball and the orbit. After puncture, the pipette was inserted to a depth of 2-4 mm behind the eyeball. Control of entering the venous plexus - filling the capillary of the pipette with blood (Dyakonov AV, Khrikina IS, Hegai AA, etc., 2013).

\section{Statistical processing of the obtained results}

To identify changes in the studied parameters (endothelial and inducible NO-synthase activity) between different groups and at different stages, we used parametric statistical methods, which are based on operating with the parameters of statistical distribution (mean and variance).

The methods used are designed for normally distributed data, so we performed a check of all data for normality using the criterion of asymmetry and excess EI Pustylnyk. According to this criterion, the distribution is different from normal if calculated empirical values of skewness and kurtosis do not exceed critical, ie $A_{e m p}<A_{\mathbf{C r}}, E_{e m p}<E_{\mathbf{C r}}$, where $A_{e m p}$ and $E_{e m p}$ - calculated values of asymmetry and excess, and

$$
A_{c r}=3 \cdot \sqrt{\frac{6 \cdot(n-1)}{(n+1) \cdot(n+3)}}, E_{c r}=5 \cdot \sqrt{\frac{24 \cdot n \cdot(n-2) \cdot(n-3)}{(n+2)^{2} \cdot(n+3) \cdot(n+5)}}
$$

respectively, their critical values [15].

All the data that we are considering was revealed by normal rosetting, so you can compare the Average values of vibroscopes in pairs. Note that in further comparisons, we perform comparisons in independent samples. These will be comparisons between different groups of animals or a comparison between that there is a group of animals (but since the sample does NOT have correspondences between animals, they will also be Independent).

Before comparing the averages of the two samples, it should be determined whether the variances are homogeneous. For this purpose it is necessary to carry out check for homosketicity (homogeneity of dispersions).

Statistical hypotheses are as follows:

$\mathbf{H}_{0}$ : the variance in group 1 does not differ from the variance in group 2.

$\mathbf{H}_{1}$ : the variance in group 1 is greater than the variance in group 2.

Hypotheses in the criterion are directed, so the criterion is one-sided. Hypothesis $\mathrm{H}_{0}$ is rejected when $F_{e m p}>F_{c r}$. This is evidenced by the p-value - the probability of error to 
reject the null hypothesis when it is correct. In various experiments, take $\mathrm{H}_{0}$ when p-value> ( significance level set), and reject $\mathrm{H}_{0}$ when $\mathrm{p}$-value <. In all subsequent calculations, we chose a standard level of significance $=0.05$.

The comparison of averages is performed using Student's t-test. When comparing the average directed hypotheses will be as follows:

$\mathbf{H}_{\mathbf{0}}$ : the average of group 1 does not differ from the average of group 2.

$\mathbf{H}_{1}$ : the average of group 1 is greater than the average of group 2.

To make a decision, the absolute value of the calculated $t$ is compared with a onesided critical. If $\left|t_{e m p}\right|<t_{c r}$, then the null hypothesis cannot be rejected. Here it is similarly possible to draw a conclusion and on p-value.

We will perform all tests in the statistical package PASW Statistics 18. We will use the t-test procedure for independent samples, which immediately compares variances and means.

\section{The results of the study and their discussion:}

A necessary link in antioxidant protection is a group of enzymes that neutralize hydrogen peroxide. One such enzyme is peroxidase [16]. This enzyme is able to catalyze oxidase and peroxidase oxidation and thus is able to take an active part in many chemical reactions of the body [17]. Peroxidase is able to control the level of hydrogen peroxide by reducing it to water [16]. It is a two-component enzyme class oxyoreductase, which consists of hematin and apoenzyme [17]. It belongs to a large group of enzymes that channel the oxidation reactions of inorganic and organic substrates using organic peroxides as electron acceptors or hydrogen peroxide [16]:

$$
\begin{aligned}
& 2 \mathrm{XH}+\mathrm{H} 2 \mathrm{O} 2 \rightarrow 2 \mathrm{X}+2 \mathrm{H} 2 \mathrm{O} ; \\
& 2 \mathrm{XH}+\mathrm{ROOH} \rightarrow 2 \mathrm{X}+\mathrm{H} 2 \mathrm{O}+\mathrm{ROH},
\end{aligned}
$$

where XH - restored substrate, $\mathrm{X}$ - oxidized substrate

Peroxidase is a link in the electron transfer chain in the mitochondrial alternative respiratory chain. It participates in redox reactions of photosynthesis, reduction of nitrates and nitrites of nitrogen metabolism, respiratory processes, participates in the regulation of development and organogenesis [16, 18].

In the cell, peroxidases play an important role in maintaining molecules in a restored state, which is one of the keys to homeostasis. Peroxidases belong to inducible enzymes, which under the influence of various factors can change their lysoforms or increase the activity of 
existing molecular forms [19]. The results of the study of peroxidase activity in the conditions of our experiment are presented in Table 1.

Table 1. - The level of peroxidase in the blood of experimental animals with simulated diabetic retinopathy and with different methods of its correction on the 30th, 60th and 180th day $(\mathrm{M} \pm \mathrm{m})$

\begin{tabular}{|l|c|c|c|}
\hline \multicolumn{1}{|c|}{ Stages } & I & II & III \\
\hline Groups & & & $152,1 \pm 3,02$ \\
\hline group & $152,1 \pm 2,63$ & $152,1 \pm 3,89$ & $86,9 \pm 3,22$ \\
\hline group & $101,89 \pm 1,88$ & $92,3 \pm 3,43$ & $112,1 \pm 3,15$ \\
\hline 3 group & $104,3 \pm 2,92$ & $108,9 \pm 2,57$ & $124,6 \pm 2,43$ \\
\hline 4 group & $112,4 \pm 3,1$ & $121,7 \pm 2,86$ & $120,1 \pm 2,74$ \\
\hline 5 group & $109,89 \pm 2,26$ & $114,2 \pm 3,08$ & $132,3 \pm 3,58$ \\
\hline 6 group & $124,7 \pm 3,08$ & $129,8 \pm 2,96$ & $150,6 \pm 3,05$ \\
\hline 7 group & $134,2 \pm 2,63$ & $141,9 \pm 3,11$ & \\
\hline
\end{tabular}

In the second group, in which the pathological process was not corrected, a decrease in this marker by $49.28 \%$ ( $p<0.001$ ) relative to the norm at the first stage was revealed. At the second stage, the activity of the indicator is $64.78 \%$ lower $(\mathrm{p}<0.001)$ compared with the data of intact animals and by $10.39 \%(\mathrm{p}<0.01)$ relative to the results of the first stage. At the third stage, the activity of this marker is $75.03 \%$ ( $\mathrm{p}<0.001$ ) lower than in the intact group. Compared with the first stage, there is a decrease of $17.25 \%(\mathrm{p}<0.001)$.

In group No. 3, it was found that at the first stage, the peroxidase activity was lower by $45.83 \%$ ( $\mathrm{p}<0.001$ ) compared with the $1^{\text {st }}$ group. No differences were found in relation to group \# 2. At the second stage, the difference from the intact group was $39.67 \%$ (p<0.001) towards a decrease in activity, and compared with group No. 2, the activity was $15.24 \%$ higher $(\mathrm{p}<0.001)$. At the third stage, the enzyme activity slightly increased - by $6.96 \%$ ( $\mathrm{p}<0.05)$ compared with the first stage. It is lower by $35.68 \%(\mathrm{p}<0.001)$ relative to the intact group, and by $22.48 \%$ (p <0.001) higher relative to group No. 2.

In the 4th group, at the first stage, the peroxidase activity is $35.33 \%$ lower $(\mathrm{p}<0.001)$ compared with group No. 1, and compared with groups No. 2 and No. 3, an improvement of $9.35 \%$ is observed $(\mathrm{p}<0,01)$ and $7.2 \%(\mathrm{p}<0.05)$, respectively. At the second stage, the enzyme activity is $7.64 \%$ higher $(\mathrm{p}<0.05)$ compared to the previous one. Relative to group No. 1 , it is lower by $24.97 \%$ ( $\mathrm{p}<0.001$ ), and compared with the results of groups 2 and 3, it is higher by $24.16 \%(\mathrm{p}<0.001)$ and $10.52 \%$ ( $\mathrm{p}<0.001)$ respectively. At the third stage, in comparison with the first, the result is the best by $9.79 \%$ ( $p<0.01$ ). In comparison with the 
data of the intact group, the activity of the marker is reduced by $22.07 \%$ ( $\mathrm{p}<0.001)$. In relation to the $2^{\text {nd }}$ group, the activity of the marker is higher by $30.26 \%(\mathrm{p}<0.001)$, and in comparison with group No. 3 - by $10.03 \%$ ( $p<0.01$ ).

In the fifth group, on the thirtieth day of the experiment, the activity of the marker is $38.41 \%$ ( $\mathrm{p}<0.001$ ) lower than in the intact group. In comparison with the $2^{\text {nd }}$ group, the activity is $7.28 \%$ higher ( $\mathrm{p}<0.01$ ). No statistically significant differences were found with respect to the $3^{\text {rd }}$ and $4^{\text {th }}$ groups. At the second stage (the sixtieth day of the study), the marker activity is $33.18 \%$ lower ( $\mathrm{p}<0.001$ ). Poriviano with group 2 , the activity is less reduced by $19.18 \%$ ( $\mathrm{p}<0.001$ ). No statistically significant differences were found with respect to the $3^{\text {rd }}$ group. And in comparison with group No. 4, the peroxidase activity is lower by $6.57 \%$ ( $\mathrm{p}<0.05)$. At the third stage, the activity is $8.5 \%$ higher $(\mathrm{p}<0.01)$ compared to the first stage. With respect to the intact group, the level of activity is lower by $26.64 \%$ ( $p<0.001$ ), and compared with the $2^{\text {nd }}$ group - higher by $27.64 \%$ ( $p<0.001$ ). Compared with group No. 3 , the activity of the marker is higher by $6.66 \%(\mathrm{p}<0.05)$. No statistically significant differences were found with respect to group 5 .

In the sixth group, according to the first stage, the activity of lower peroxidases was $21.98 \%$ ( $p<0.001$ ) compared with group No. 1, and by $18.29 \%$ ( $<<0.001$ ) compared with group No. 2. With respect to all subsequent groups, in which the modeled pathology was corrected, the marker activity in the 6th group was higher: by $16.36 \%(\mathrm{p}<0.001)$ compared to the third group, by $9.86 \%$ ( $\mathrm{p}<0.01$ ) compared from the 4th, and by $11.87 \%$ ( $\mathrm{p}<0.001$ ) compared with group No. 5. At the second stage, the value of the indicator was lower by $17.18 \%$ ( $\mathrm{p}<0.001)$ compared with group No. 1 . Relative to the $2^{\text {nd }}$ group, the activity is higher by $28.89 \%$ ( $p<0.001$ ), relative to the 3 rd - by $16.1 \%$ ( $p<0.001)$, comparable to the $4^{\text {th }}$ and $5^{\text {th }}$ groups, the activity was also not painful - by $6.24 \%(\mathrm{p}<0.05)$ and $12.02 \%(\mathrm{p}<0.001)$, respectively. At the third stage, the activity of the marker is $14.96 \%(\mathrm{p}<0.001)$ of the lower relative to the intact group. Compared with the $2^{\text {nd }}$ group, the activity is $34.32 \%$ higher ( $\mathrm{p}<0.001)$, compared with the third - by $15.27 \%$ (p <0.001), compared with the $4^{\text {th }}$ - more by $5.82 \%(\mathrm{p}<0.05)$, and in relation to the $5^{\text {th }}-$ by $9.22 \%(\mathrm{p}<0.01)$.

In group No. 7 , at the first stage, the activity of the lower one by $13.34 \%(\mathrm{p}<0.001)$ relative to the intact group of animals. The threshold value with the $2^{\text {nd }}$ group of increased activity activity by $24.07 \%$ ( $\mathrm{p}<0.001$ ), the threshold value with the third group - by $22.28 \%$ ( $\mathrm{p}<0.001)$, in the threshold value with the $4^{\text {th }}$ group - by $16,25 \%(\mathrm{p}<0.001)$. Relative to the $5^{\text {th }}$ group, the activity is the best by $18.11 \%(\mathrm{p}<0.001$ ), and in relation to the 6 th - by $7.08 \%$ ( $p<0.05)$. At the second level, the identification of the marker activity by $5.43 \%(p<0.05)$ is 
equal to the $30^{\text {th }}$ day. Relative to the intact group, the activity is lower by $7.18 \%$ ( $\mathrm{p}<0.05$ ), but with group No. 2 it is higher by $34.95 \%$ ( $\mathrm{p}<0.001$ ). There is a positive trend in comparison with all groups in which the pathological process was corrected: the peroxidase activity is higher by $23.26 \%$ ( $\mathrm{p}<0.001$ ) compared to the third group, by $14.24 \%$ ( $\mathrm{p}<0.001)$ compared to the fourth, by $19.52 \%(\mathrm{p}<0.001)$ in the defect with group No. 5 and by $8.53 \%$ ( $\mathrm{p}<0.01)$ in the defect with the sixth. At the third stage of the study, the normal activity of the study of the indicator was found: by $10.89 \%$ ( $\mathrm{p}<0.001$ ) equally with the indicated value $5.77 \%(\mathrm{p}<0.05)$ was generated from the second, collected groups created earlier (which certainly indicates efficiency of correction). Peroxidase activity with group No. 2 is higher by $42.30 \%$ ( $\mathrm{p}<0.001$ ). Also, a more pronounced positive effect was found in porrivianni in all preliminary groups in which DR was carried out: by $25.56 \%(\mathrm{p}<0.001)$ relative to the third group, by $17.26 \%$ (p <0.001) - relative to the fourth by $2025 \%$ (p<0.001) equally with the fifth group and $12.15 \%$ ( $\mathrm{p}<0.001)$ equally with the sixth.

We can state the effectiveness of the application of the correction method in the 6th group for the normalization of peroxidase activity, although the effectiveness is not one hundred percent. In group No. 7, more pronounced positive results were found - the activity of the indicator increased to normal values.

The results of studying the peroxidase activity under experimental conditions are clearly illustrated in Fig. 1.

(A) Box-plots illustrate the distribution of peroxidase activity values in each group at the first stage of the study ( $\mathrm{n}=20$ in each of the study groups). (B) Box-rafts illustrate the distribution of peroxidase activity values in each group at the second stage of the study ( $\mathrm{n}=$ 20 in each of the study groups). (B) Box-plots illustrate the distribution of peroxidase activity values in each group at the third stage of the study ( $\mathrm{n}=20$ in each of the study groups) 
A

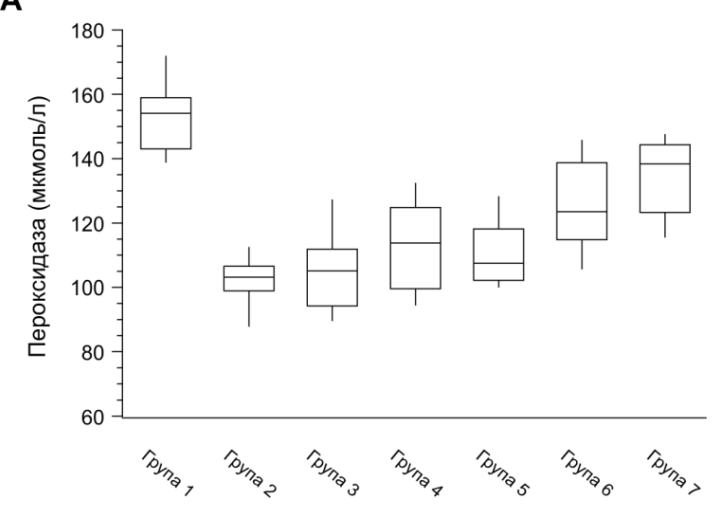

B

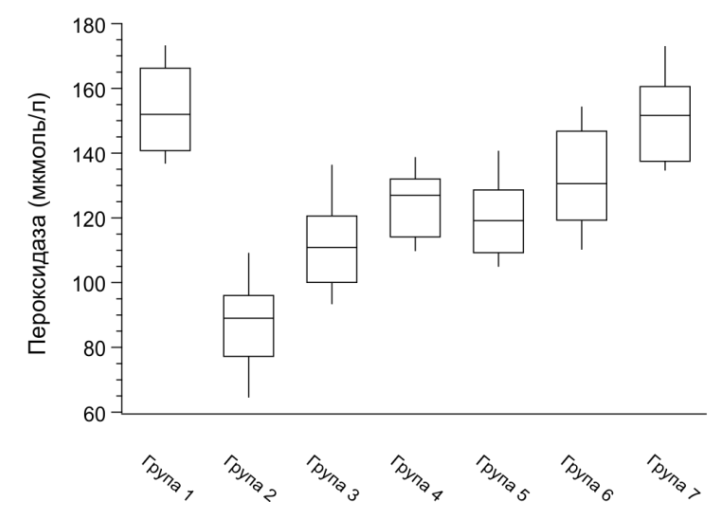

Б

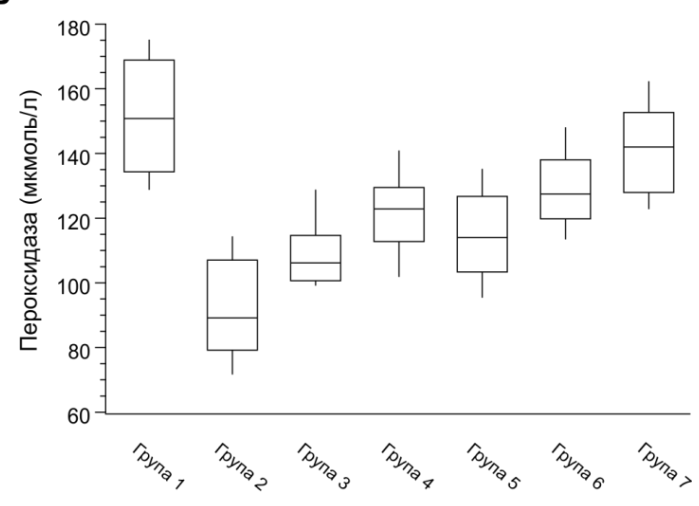

Figure 1 - Change in peroxidase activity in each of the experimental groups on the $30^{\text {th }}, 60^{\text {th }}$ and $180^{\text {th }}$ day after modeling diabetic retinopathy

\section{Conclusions:}

1. The results indicate the development of oxidative stress, starting from the 30th and with subsequent progression on the 60th and 180th days of experimental diabetic retinopathy, which is confirmed by a decrease in peroxidase activity in the 2nd group, the maximum of which is observed at 3- th stage.

2. Correction with hypoglycemic agents in group 3 had a positive effect, but was not able to restore the activity of the antioxidant enzyme, so there was a need for additional drugs.

3. The use of aflibercept and nitric oxide donor in group 4 to correct the development of diabetic retinopathy had a positive effect on increasing the activity of peroxidase, the maximum of which occurred on the 180th day of the experiment, but did not reach the control values.

4. It was proved that the combined administration of bromfenac and aflibercept in group 5 significantly increased the antioxidant activity, but not as significantly as in group 4 . 
5. It was proved that the administration of aflibercept, L-carnitine and bromfenac to animals of the 6th group restored antioxidant protection on the 30th and was continued on the 60th and 180th day of the study, but the results did not reach the control values.

6. The most effective correction was a combination of metformin, aflibercept, Larginine and citicoline in rats of group 7, as evidenced by the normalization of peroxidase activity on the 30th and 60th day of the experiment, and on the 180th was recorded restoration of marker activity to control indicators.

\section{List of references}

1. Endothelial dysfunction in the pathogenesis of complications of diabetes Message I. Endothelial dysfunction: etiology, pathogenesis and diagnostic methods AI Gozhenko, G.S. Kuznetsova, K.S. Kuznetsova, T.M. Beat, A.B. Wort. Endocrinology. 2017, T 22, No. 2. S. 171-181.

2. Kurkin DV, Logvinova EO, Bakulin DA, Volotova EV, Tyurenkov IN Endothelioprotective properties of a new agonist of the gpr119 receptor (dipiaron) in animals with chronic cerebral circulatory disorders and experimental diabetes mellitus // Modern problems of science and education. - 2018. - № 4 ; URL: http://www.scienceeducation.ru/ru/article/view?id=27935

3. Expression modification of uncoupling proteins and MnSOD in retinal endothelial cells and pericytes induced by high glucose: the role of reactive oxygen species in diabetic retinopathy / Y. Cui [et al.] // Exp. Eye Res.— 2006.— Vol. 83, № 4.— P. 807-816.

4. Efficiency of use of cryopreserved mesenchymal placental stem cells in the correction of oxidative status in type 2 diabetes mellitus / Yu. A. Demin, M. Yu. Demina / International Medical Journal, 2015, № 3.S.55-58.

5. Oxidative stress in diabetes: implications for vascular and other complications / D. Pitocco, M. Tesauro, R. Alessandro [et al.] // Int. J. Mol. Sci._ 2013._ Vol. 14.— P. 2152521550.

6. Kowluru R. A. Effect of reinstitution of good glycemic control on retinal oxidative stress and nitrative stress in diabetic rats / R. A. Kowluru // Diabetes. - 2003. - Vol. 52, № 3.- P. 818-823.

7. Zhong Q. Epigenetic changes in mitochondrial superoxide dismutase in the retina and the development of diabetic retinopathy / Q. Zhong, R. A. Kowluru // Diabetes.2011.— Vol. 60, № 4.- - P. 1304-1313. 
8. Oxidative stress in type 1 diabetes / K. Haskins [et al.] // Ann. of the New York Academy of Sciences. — 2003.— Vol. 1005.— P. 43-54.

9. Pasechnikova NV Protective action of quercetin and lipoate on functional groups of retinal proteins in modeling diabetes / NV Pasechnikova. Pasechnikova, O.A. Moroz // Ophthalmological Journal. - 2015. - № 3. - P. 76-81

10. The role of metformin in the prevention of diabetic nephropathy in experimental type 2 diabetes mellitus / V.K. Bayrasheva, A. Yu. Babenko, Yu.V. Dmitriev, AA Bayramov, SG Chefu, I.S. Shatalov, A.N. Arefieva, I.Yu. Pchelin, N.V. Khudyakova, P.G. Aliev, EN Grineva // Regional blood circulation and microcirculation. - 2016. - №15 (3). - pp.70-80.

11. Pokrovsky MV Endothelioprotective effects of L-arginine in modeling nitric oxide deficiency / M.V. Pokrovsky, TG Pokrovskaya VI Korchakov // Experimental and clinical pharmacology. - 2008. - № 71 (2). - pp. 29-31.

12. Efficacy of Subconjunctival Aflibercept Versus Bevacizumab for Prevention of Corneal Neovascularization in a Rat Model / Orly Gal-Or 1, Eitan Livny, Ruti Sella, Yael Nisgav, Dov Weinberger, Tami Livnat, Irit Bahar // Cornea. - 2016. - Vol. 3. - Issue 7. - pp. 991-996.

13. Bykov I.L. Influence of L-carnitine on metabolic disorders in experimental deficiency of acyl-CoA dehydrogenases / I.L. Bykov // Experimental and Clinical Pharmacology. -2004. -Volume 67 - No. 6. P.48-52.

14. Dzugkoev SG Influence of coenzyme Q 10, afobazole and L-carnitine on endothelial function in rats with experimental diabetes mellitus. Dzugkoev, F.S. Dzugkoeva, N.V. Gumanova, V.A. Metelskaya // Kuban Scientific Medical Bulletin. - 2012. - No. 3 (132). - S.48-51.

15. Lupan IV, Avramenko OV, Akbash K.S. Computer statistical packages: a basic methodical guide. - 2nd type. - Kirovohrad: "CODE" 2015. - 236 p.

16. Kobrinets L.A. Change in the activity of enzymes of the antioxidant system in lupine seedlings caused by the action of lead compounds. Bulletin of the Brest State Technical University. 2012. No. 2 P.86-89.

17. Rogozhin, V.V. Peroxidase as a component of antioxidant systems of living organisms / V.V. Rogozhin. - SPb. : GIORD, 2004 .-- 240 p.

18. Andreeva, VA Enzyme peroxidase: participation in the defense mechanism of plants / VA Andreeva. - M .: Nauka, 1988. - P. 7-24.

19. Peroxidases as markers of plant resistance to a pathogen. Minova, A. L. Kabachevskaya, E. M. Lyakhnovich, G. V. Volotovsky, I. D. Molecular, membrane and 
cellular bases of the functioning of biosystems: Intern. scientific. conf .; Tenth Congress of the Belarusian Public Association of Photobiologists and Biophysicists, June 19-21, 2012, Minsk, Belarus: Sat. Art .: at 2 pm Part $2 /$ editorial board: I.D. Volotovsky, S.N. Cherenkevich [and others]. - Minsk: Ed. BSU Center, 2012. - pp. 128-130 